\title{
Childhood sexual abuse and obesity
}

\author{
T. B. Gustafson and D. B. Sarwer
}

University of Pennsylvania School of Medicine, Department of Psychiatry, Weight and Eating Disorders Program, Philadelphia, PA, USA

Received 15 March 2004; revised 14 May 2004; accepted 17 May 2004

Address reprint address: David B. Sarwer, PhD, University of Pennsylvania School of Medicine, Weight and Eating Disorders Program, 3535 Market Street, Suite 3108, Philadelphia, PA 19104, USA.

E-mail: dsarwer@mail.med.upenn.edu

\begin{abstract}
Summary
The causes of the current obesity epidemic are multifactorial and include genetic, environmental, and individual factors. One potential risk factor may be the experience of childhood sexual abuse. Childhood sexual abuse is remarkably common and is thought to affect up to one-third of women and one-eighth of men. A history of childhood sexual abuse is associated with numerous psychological sequelae including depression, anxiety, substance abuse, somatization, and eating disorders. Relatively few studies have examined the relationship between childhood sexual abuse and adult obesity. These studies suggest at least a modest relationship between the two. Potential explanations for the relationship have focused on the role of disordered eating, particularly binge eating, as well as the possible 'adaptive function' of obesity in childhood sexual abuse survivors. Nevertheless, additional research on the relationship between childhood sexual abuse and obesity is clearly needed, not only to address the outstanding empirical issues but also to guide clinical care.
\end{abstract}

Keywords: Body weight, child abuse, childhood sexual abuse, obesity.

obesity reviews (2004) 5, 129-135

\section{Introduction}

Obesity has proven very difficult to prevent and treat (1), at least in part because the aetiology is complex and not completely understood. While $40-70 \%$ of the variation in obesity has been estimated to be heritable (2), researchers argue that changes in the environment that currently promote excessive food intake and discourage physical activity have contributed to the increasing rate of obesity in Westernized countries (3). A variety of psychosocial conditions, from depression (4) to binge eating (5-7), also appear to be related to both the development and maintenance of obesity. A history of childhood sexual abuse (CSA) is another psychosocial condition that may serve as a risk factor for obesity. Some studies have suggested that CSA may be nearly as prevalent as obesity. Yet, somewhat surprisingly, researchers have paid relatively little attention to the potential relationship between the two.

This paper reviews the literature on the relationship between CSA and adult obesity. A Medical Line literature search using the terms 'obesity' and 'childhood sexual abuse' was undertaken to identify studies which have inves- tigated the relationship. The reference lists from those articles were used to obtain additional articles. The current paper begins with a discussion of the prevalence of CSA. It then briefly describes the psychological sequelae of CSA. Next, the relationship between CSA and obesity is examined. Subsequently, the major theoretical explanations for a link between CSA are explored. We conclude with a discussion of future research directions and clinical implications.

\section{The prevalence of childhood sexual abuse}

Numerous studies of clinical and non-clinical populations have provided general information on the rate of CSA in the general population. Nevertheless, accurate statistics on the prevalence of CSA are difficult to obtain. As detailed below, the majority of studies have methodological differences and shortcomings that have resulted in a wide range of estimates (8-10). We believe studies of large, community-based samples likely provide the most reliable information on the prevalence of CSA. These are briefly detailed below. 
Gorey and Leslie reviewed the results of 25 crosssectional studies of North American samples published between 1969 and 1991 (9). The estimate of the prevalence of CSA was calculated by combining the reports of over 20000 individuals. Results suggested that $22.3 \%$ of women and $8.5 \%$ of men were sexually abused during childhood.

Among women, there was an inverse correlation between the prevalence of CSA and response rate, suggesting that CSA victims were more likely than non-abused women to have participated in the studies. When samples with response rates of less than $60 \%$ were excluded from the original analysis, the rate of CSA decreased to $16.8 \%$ for women and $7.9 \%$ for men. When studies that included non-contact sexual abuse, such as indecent exposure, were excluded (a definitional controversy in the literature discussed below), $14.5 \%$ of women and $7.2 \%$ of men reported a history of CSA.

Three subsequent studies that relied on large, nationally representative, community-based samples found CSA in $11.1 \%$ to $32.0 \%$ of women and $3.9 \%$ to $14.2 \%$ of men (11-13). Perhaps the largest single investigation, the Adverse Childhood Experiences (ACE) study, did not use a nationally representative sample. Using a sample of 13177 residents of the San Diego, California area, sexual abuse was reported by $21.7 \%$ of respondents (14). Separate rates for males and females were not reported.

Ideally, these studies would have generated a consensus on the prevalence of CSA. Unfortunately, methodological concerns make interpretation of the findings difficult (8). One major problem is the lack of agreement within the professional community on a precise definition of abuse. While some studies have used rather specific definitions, such as defining abuse based on the occurrence of physical contact, others have used rather broad descriptions, which encompass incidents such as indecent exposure by a stranger. Furthermore, the definition of 'childhood' and the requirement of an age difference between the abuser and victim (used in an attempt to exclude individuals reporting childhood sexual play) have been inconsistent across studies. These differences, together with disparities in response rates, may account for as much as half of the variability among prevalence estimates (9). In addition, most studies relied on retrospective, self-report data, without corroboration from other sources. Thus, retrospective bias, memory deterioration, and even false memories of abuse may compromise the face validity of the findings $(15,16)$. Finally, confounding variables, such as demographics, family environment, or the coexistence of multiple forms of abuse, often were not adequately characterized.

In summary, based on a comprehensive review of three decades of research, coupled with four recent investigations, $11-32 \%$ of females and $4-14 \%$ of males have been the victims of CSA $(9,11-14)$. Unfortunately, methodolog- ical problems with the majority of investigations make more precise estimates difficult, if not impossible.

\section{Psychological sequelae of childhood sexual abuse}

Childhood sexual abuse is associated with both short- and long-term psychological difficulties. Short-term problems include depression, anxiety, sleep disturbances, somatic complaints, phobic reactions, dissociation, low self-esteem, and deficits in intellectual, physical, social, and sexual development (17). Long-term correlates of CSA include many of the same conditions as well as post-traumatic stress disorder, substance abuse, sexual dysfunction, borderline personality disorder, and suicidality (17-20).

Sexually abused women are more likely than non-abused women to receive a psychiatric diagnosis during their lifetimes $(13,21)$. The prevalence rates of 19 psychiatric diagnoses were higher among 52 adult women who had been sexually abused as children as compared to a sample of age- and race-matched controls without a history of abuse (21). More severe forms of sexual abuse were associated with higher rates of psychiatric disorders. More than half of all women $(56 \%)$ and $47 \%$ of men with a history of CSA were found to have 'any psychiatric disorder' (13). This was significantly greater than the $32 \%$ of women and $34 \%$ of men without abuse histories who were found to have at least one psychiatric diagnosis. Among women, a history of CSA was associated with higher rates of every disorder studied, including anxiety, depression, alcohol and drug abuse/dependence, and antisocial behaviour (13). Certain characteristics appear more strongly associated with psychological sequelae than others, including sexual abuse at a later age, a greater number of abuse incidents or involvement of multiple abusers, abuse involving oral, anal or vaginal penetration, and a greater level of emotional upset at the time of abuse (11).

Disordered eating is another common psychological sequale of CSA. It also may be the most relevant one to the relationship between CSA and obesity. A recent metaanalysis of 53 studies of the relationship between CSA and eating disorders concluded that a small, significant positive relationship exists between the two (22). Estimates of effect size of the relationship, however, ranged from 0.12 to 0.28 and varied based on the nature of the sample, the definitions of abuse employed, and whether CSA or eating disorder status was used as the independent variable in the analysis.

Specific investigations of anorexia nervosa and bulimia nervosa have produced heterogeneous results. A recent review (23) concluded that CSA was found to be a nonspecific risk factor for bulimia nervosa, but not for anorexia nervosa. Ten out of 15 studies included suggested a positive correlation between CSA and bulimia nervosa. Of the six studies that examined both disorders, four found 
that bulimia nervosa was diagnosed more frequently than anorexia nervosa in CSA victims; the other two studies found no difference in rates of diagnosis. In addition, CSA appeared to be associated with heightened levels of psychiatric comorbidity in eating disordered individuals, but not with the severity of the eating disturbance.

As with the research on the prevalence of CSA, several methodological concerns exist with studies of the sequelae of sexual abuse (24). In addition to the problems in defining sexual abuse detailed above, these studies typically have failed to include appropriate control groups or the most valid and reliable assessments of psychiatric symptoms or formal psychopathology. Many studies have included only samples drawn from psychiatric populations, which may explain the high rates of psychopathology found in several studies (25).

In summary, despite methodological shortcomings of the literature, the experience of CSA appears to be associated with a range of adult psychiatric conditions. The strongest relationships appear with general psychological distress, depression, anxiety, substance abuse, and somatization. The relationship with disordered eating is somewhat less strong. A history of CSA appears to be more strongly associated with bulimia nervosa than anorexia nervosa. This finding leads to the intuitive question of the possible relationship between CSA and adult obesity.

\section{Childhood sexual abuse and obesity}

There has been little investigation of the association between CSA and obesity. The first study to examine this relationship was prompted by the clinical observation that CSA seemed to be surprisingly common among obese patients enrolled in a weight loss programme (26). A chart review of 131 patients revealed that $60 \%$ of those who reported a history of rape or sexual molestation were $\geq 50$ pounds overweight, as compared to only $28 \%$ of persons matched on sex and age, but without a history of abuse. CSA victims were also more likely to suffer from extreme obesity. Twenty-five per cent of the abused group was found to be $\geq 100$ pounds overweight, compared with only $6 \%$ of controls. Based on information extracted from patients' medical records, the abuse typically preceded and was often proximate to the onset of obesity.

Subsequent investigations have provided additional evidence for a relationship between CSA and obesity. Among 511 female, family practice patients in a rural community in the Midwestern United States, those with a history of CSA were more likely to be overweight (defined as $40 \%$ or more above ideal body weight) and to report having ever been overweight, compared with non-abused controls (27). Felitti further investigated the link between abuse and obesity by comparing the experiences of 100 significantly overweight patients (defined as 65 pounds over the heavy frame
Metropolitan Insurance Company ideal body weight) enrolled in a weight loss programme to 100 always-slender controls (28). The overweight patients, compared to normal-weight patients, were more likely to have a history of CSA $(25 \%$ vs. $6 \%)$. They were also found to have more frequent histories of non-sexual childhood abuse (29\% vs. $14 \%)$, loss of parent as infant, child, or adolescent $(48 \%$ vs. $23 \%$ ), and parental alcoholism $(40 \%$ vs. $17 \%)$. These results suggest that adverse childhood experiences, including, but not limited to sexual abuse, are associated with adult obesity.

The Adverse Childhood Experiences (ACE) study has further investigated the association between a variety of adverse childhood experiences and physical or mental health problems in later life. Following an initial evaluation, participants were mailed questionnaires asking about three categories of abuse (recurrent physical abuse, recurrent severe emotional abuse, and sexual abuse) and five categories of household dysfunction (growing up in a household where someone was in prison; where the mother was treated violently; with an alcoholic or drug user; where someone was chronically depressed, mentally ill, or suicidal; and where at least one biological parent was lost to the patient during childhood, regardless of cause). Those who experienced four or more categories of childhood abuse or household dysfunction had 1.4- to 1.6-fold increased risk of having a body mass index (BMI) $\geq 35 \mathrm{~kg} \mathrm{~m}^{-2}$ compared to those who had experienced no abuse or dysfunction (29).

After controlling for a variety of psychosocial factors, including household dysfunction in childhood, smoking, alcohol, physical activity, education, employment status, and parity, statistically significant increases in body weight were seen for respondents who reported severe sexual abuse (14). Specifically, participants who reported a history of abuse that consisted of 'touched and attempted intercourse' were an average of $1.4 \mathrm{~kg}$ [95\% confidence interval $(\mathrm{CI})=0.2-2.5 \mathrm{~kg}$ ] heavier than those who denied sexual abuse. Individuals who reported abuse that involved intercourse weighed an average of $2.2 \mathrm{~kg} \quad(95 \% \quad \mathrm{CI}=1.2-$ $3.2 \mathrm{~kg}$ ) more than those who did not report past sexual abuse. Although statistically significant, these differences represent a relatively small percentage of the average respondent's total body weight (mean weight for entire sample $=78.0 \pm 17.5 \mathrm{~kg}$ ). Nevertheless, sexual abuse that involved any type of penetration was associated with an increased risk of obesity (relative risk $=1.29$, 95\% $\mathrm{CI}=1.16-1.45)$. Body weights and risks of obesity in those reporting less severe forms of sexual abuse also were elevated, although not statistically significant.

Other studies have found a relationship between CSA and extreme obesity. The National Women's Study conducted structured telephone interviews on a nationally representative sample of 3012 American women and inquired 
about experiences of sexual trauma and harassment and a variety of medical and psychiatric conditions (30). A self-reported history of child or adult sexual abuse was associated with a lifetime diagnosis of extreme obesity $\left(\mathrm{BMI}>40 \mathrm{~kg} \mathrm{~m}^{-2}\right)(31)$. Unfortunately, this study provided little additional information on the relationship between unwanted sexual experiences and body weight.

In contrast, one study found no relationship between CSA and obesity. In an investigation of 150 patients who presented for routine gynaecological care, $26 \%$ reported a history of sexual abuse. Those with and without a history of sexual abuse did not differ in BMI. This study, however, included only 13 obese women with a history of sexual abuse, which may have resulted in insufficient statistical power to identify differences in BMI across abuse status groups (32).

Results from the ACE study and National Women's Study provide some of the most compelling information on the relationship between sexual abuse and obesity. The large sample sizes and breadth of data collected are significant methodological strengths. Nevertheless, both studies have methodological limitations. First, similar to most of the CSA literature, these studies relied on retrospective, self-report data collected often decades after the occurrence of the abuse. Second, the ACE study has somewhat limited generalizability as it examined only patients from a Health Maintenance Organization in San Diego, California who were evaluated at a health appraisal clinic. As $80 \%$ of participants were self-referred for medical care, there also is some concern about selection bias.

Most of the evidence to date suggests a small, positive correlation between CSA and obesity. This relationship appears to be particularly strong in cases of more severe sexual abuse. Despite efforts made to control for potential confounds, the correlational nature of the findings makes it difficult to make definitive statements about a potential causal relationship between CSA and obesity.

\section{Possible explanations of the CSA-obesity relationship}

Researchers have suggested several possible explanations for the apparent association between CSA and obesity. These include the hypothesis that binge eating may serve as a mediating variable between CSA and obesity as well as the idea that obesity may serve an 'adaptive function' in some women with a history of CSA.

\section{Binge eating as a mediator of the relationship between CSA and obesity}

Numerous studies have documented a strong relationship between binge eating and obesity (5-7). While binge eating would be thought to usually precede the development of obesity, few studies have identified such a temporal relationship. It is equally plausible that binge eating contributes to not only the development, but also the maintenance of obesity.

Several studies have suggested a relationship between CSA and the diagnosis of binge eating disorder (BED). In a sample of 145 consecutive outpatients with BED, a history of sexual abuse was found in $30.3 \%$, compared to only $18.4 \%$ of normal controls (33). Similarly, in a community sample of 162 women with BED, $35.3 \%$ reported a history of CSA, significantly greater than the $12.2 \%$ among controls (34).

In contrast, two studies failed to establish a relationship between CSA and BED. One of these studies, however, was an investigation of extremely obese, treatment-seeking individuals with a high rate of BED (>60\%), with only a limited number of sexually abused individuals $(n=22)(35)$. The other study was an investigation of 20 sexually abused adolescents (36). The unique characteristics and small sizes of these two samples could account for the lack of positive findings.

Wonderlich has suggested that the relationship between CSA and disordered eating may be mediated by several variables, including mood, behavioural impulsivity, body image disturbance, drug use, and poor self-esteem. Alternatively, a traumatic experience such as sexual abuse may result in psychobiological dysregulation, which may negatively influence maladaptive behaviours, including disordered eating $(23,36)$. CSA or similar traumas may cause disturbances in the 5-hydroxytryptamine [5-HT] pathway (37-39), which has been shown to play a key role in the regulation of eating behaviour (40-42). The role of 5-HT in the development of obesity, however, awaits further study.

In summary, several studies have suggested that CSA is positively correlated with BED $(33,34)$. Given the wellestablished relationship between BED and obesity, coupled with thought-provoking findings suggesting a relationship between reduction in 5-HT activity and childhood trauma (39) as well as binge eating (42), it is plausible that the relationship between CSA and obesity is mediated by binge eating. Clearly, this hypothesis awaits further investigation in future studies.

\section{Obesity as an 'adaptive function'}

Some investigators have proposed that in some subset of obese women who have a history of sexual abuse, obesity serves an 'adaptive function' as a self-protective mechanism (32). Sexually abused women experience significantly increased rates of sexual dysfunction $(19,43)$ and also are at greater risk for subsequent victimization (17). As thinness is one component of current Western ideals of female beauty and sexuality $(44,45)$, some female sexual abuse survivors may experience their obesity as a form of protec- 
tion from sexual advances by potential sexual partners and/ or abusers $(28,32,46,47)$. Alternatively, others may not experience the same degree of dissatisfaction with their weight and body image that may otherwise motivate a weight loss effort (48).

For some women with a history of sexual abuse, weight reduction may trigger post-traumatic stress disorder symptoms as women approach the weight at which they were abused $(35,49)$. Weiner and Stephenson have suggested that some women may have 'barrier weights' (50). These are body weights, usually lower than the individuals' current weights, which if approached, may produce a great deal of anxiety. Some female eating-disorder patients have been described as avoiding specific body weights that correspond to events in their sexual history. Methodological limitations with the study, including a highly selective sample of eatingdisorder patients and lack of statistical analyses, lead to some question about these findings. Nevertheless, the idea of women having a 'barrier weight' related to sexual victimization has some intuitive appeal.

Finally, obesity may serve a 'positive' role within some stable romantic relationships. Approximately one-fifth $(22 \%)$ of obese patients using a very-low-calorie diet (VLCD) acknowledged being aware of using their obesity to decrease sexual fears (28). As these individuals perceived themselves as less physically attractive to the opposite sex because of their obesity, they worried less about spousal jealousy and their own marital fidelity. Interestingly, for some, these concerns do not appear to be imagined; $13 \%$ of the obese group reported an increase in spousal jealousy as they lost weight. Intuitively, some CSA victims may be more sensitive to sexual fears than non-abused individuals, and therefore more likely to benefit from any reduction in these fears that their obesity provides. This idea, however, has yet to be empirically investigated.

Researchers also have examined the notion of obesity serving an 'adaptive function' in CSA victims by looking for correlations between abuse history and weight loss treatment failure. Among obese women enrolled in a weight loss programme using a VLCD, those with a history of sexual abuse lost significantly less weight and reported more episodes of non-adherence, compared with nonabused women (35). Similarly, among 190 obese patients who each lost over 100 pounds in a VLCD programme, those with a history of CSA had a significantly higher likelihood of weight regain at 18 months (51). Mediating variables between CSA and these weight loss failures may include immature coping styles (52) and behavioural impulsivity (53), both of which are seen more commonly in abuse victims and, intuitively, could contribute to decreased success in weight loss programmes that require high levels of self-control.

Weiderman, Sansone, and Sansone further explored the tendency toward weight-loss failure seen in CSA victims and found that obese sexual abuse victims reported less current body dissatisfaction and less weight fluctuation during adulthood compared with non-abused obese women (32). When asked about weight loss expectations, the vast majority of women reported that they desired weight losses that would bring their BMI into the 'normal' range. Only three women reported an 'ideal weight' which remained in the obese range; all three were abuse survivors. These findings led the authors to argue that at least some obese women with a history of CSA find their obesity more acceptable than do non-abused, obese women.

In summary, several authors have suggested that for some subset of obese CSA survivors, obesity may serve as an 'adaptive function' which may protect against unwanted romantic or sexual interest. Preliminary results suggest that compared to non-abused individuals, CSA survivors appear to be less dissatisfied with their obesity and less likely to be successful at obtaining and maintaining weight loss.

\section{Conclusions and future directions}

As the obesity epidemic has developed, increased empirical attention has been paid to both the environmental and psychosocial factors associated with the disorder. One factor that has received only limited attention to date has been a history of sexual abuse. Several studies have identified a relationship between a history of CSA and obesity. The relationship appears to be particularly strong for those women who experienced more severe forms of abuse, such as incidents involving penetration. Although the exact mechanism of the relationship is yet unknown, and likely multifactorial, at least two hypotheses with intuitive appeal have been proposed. Given the relationship of disordered eating to both CSA and obesity, it is possible that maladaptive eating behaviours mediate the two. It also has been suggested that obesity serves an 'adaptive function' in some abuse victims, as a means of protection from experiences that may trigger adverse emotional experience related to the abuse.

Clearly, additional research on the relationship between CSA and obesity is needed. Perhaps the most significant challenge for future studies is to avoid many of the methodological pitfalls of the previous investigations. Subsequent studies should rely upon reasonable definitions of sexual abuse widely used in previous research. Similarly, studies should strive for large, heterogenous sample sizes and include appropriate comparison or control groups. As with all investigations of psychosocial characteristics, researchers should rely on valid and reliable psychometric measures.

Preliminary evidence suggests that the relationship between CSA and obesity may be mediated by disordered eating. Clearly, this is an area worthy of additional study. Similarly, the idea that obesity may serve as an 'adaptive function' in some CSA victims, making weight reduction 
even more challenging, warrants further investigation. Several physiological and behavioural barriers to successful long-term weight control have been identified. Others yet to be identified likely exist as well. A history of CSA may be a particularly strong barrier to overcome for some individuals. Finally, further exploration should be carried out on the link between psychobiological dysregulation, eating behaviour, and obesity, both to understand better the pathophysiology of obesity and to investigate potential pharmacological interventions.

The current review has focused on the experiences of unwanted sexual experiences in childhood and their relationship to adult obesity. Little is known about the relationship of CSA and the development of childhood or adolescent obesity. Some evidence suggests that the sexual abuse often predates the development of obesity (26). Alternatively, the experience of being an obese child or adolescent may unfortunately represent an increased susceptibility to many forms of both sexual and non-sexual maltreatment and abuse. Long-term, prospective studies of obese children with and without a history of abuse would provide important information on the possible directional nature of the relationship between CSA and obesity.

Given this relationship, patients presenting for weight reduction should be asked about a history of sexual abuse. Such inquiry needs to be carried out in a supportive, nonjudgemental manner. The objective of this questioning is to not obligate patients to discuss a particularly painful experience in their past with the goal of resolving the issue. Rather, it may shed some light on patients' perceptions of the possible relationship between the historical abuse and their current body weight. Patients' body weight at the time of the abuse should be documented and the possibility that this weight may function as a 'barrier weight' should be considered if patients are unsuccessful with conventional weight loss efforts or experience a premature weight plateau. If patients become visibly upset when discussing a history of abuse, or if the experience of a 'barrier weight' is suspected, a referral to a mental health provider with expertise in sexual abuse is warranted.

\section{Acknowledgements}

Support for this paper was provided, in part, by a University of Pennsylvania School of Medicine FOCUS on Health and Leadership for Women Medical Student Fellowship to Dr Gustafson and by NIH/NIDDK Grant K23 DK6002303 to Dr Sarwer.

\section{References}

1. Wadden TA, Brownell KD, Foster GD. Obesity: responding to the global epidemic. J Consult Clin Psychol 2002; 70:510525 .
2. Comuzzie AG, Allison DB. The search for the human obesity genes. Science 1998; 280:1374-1377.

3. Hill JO, Peters JC. Environmental contributions to obesity epidemic. Science 1998; 280:1371-1374.

4. Onyike CU, Crum RM, Lee HB, Lyketsos CG, Eaton WW. Is obesity associated with major depression? Results from the Third National Health and Nutrition Examination Survey. Am J Epidemiol 2003; 158:1139-1147.

5. Stunkard AJ, Allison KC. Two forms of disordered eating in obesity: binge eating and night eating. Int J Obes Relat Metab Disord 2003; 27:1-12.

6. de Zwaan M. Binge eating disorder and obesity. Int J Obes Relat Metab Disord 2001; 25:S51-S55.

7. Spitzer RL, Yanovski S, Wadden T, Wing R, Marcus MD, Stunkard A, Devlin M, Mitchell J, Hasin D, Horne RL. Binge eating disorder: its further validation in a multisite study. Int J Eat Disord 1993; 2:137-153.

8. Pope H, Hudson J. Does childhood sexual abuse cause adult psychiatric disorders? Essentials of methodology. J Psychiat Law $1995 ; 23: 363-381$.

9. Gorey KM, Leslie DR. The prevalence of child sexual abuse: integrative review adjustment for potential response and measurement biases. Child Abuse Negl 1997; 21:391-398.

10. Finkelhor D. Current information on the scope and nature of child sexual abuse. Future Child 1994; 4:31-53.

11. Briere J, Elliott DM. Prevalence and psychological sequelae of self-reported childhood physical and sexual abuse in a general population sample of men and women. Child Abuse Negl 2003; 27:1205-1222.

12. Vogeltanz ND, Wilsnack SC, Harris TR, Wilsnack RW, Wonderlich SA, Kristjanson AF. Prevalence and risk factors for childhood sexual abuse in women: national survey findings. Child Abuse Negl 1999; 23:579-592.

13. MacMillan HL, Fleming JE, Trocme N, Boyle MH, Wong M, Racine YA, Beardslee WR, Offord DR. Prevalence of child physical and sexual abuse in the community. Results from Ontario Health Supplement. JAMA 1997; 278:131-135.

14. Williamson DF, Thompson TJ, Anda RF, Dietz WH, Felitti $\mathrm{V}$. Body weight and obesity in adults and self-reported abuse in childhood. Int J Obes Relat Metab Disord 2002; 26:1075-1082. 15. Loftus EF. Make-believe memories. Am Psychologist 2003; 58:867-873.

16. Maughan B, Rutter M. Retrospective reporting of childhood adversity: issues in assessing long-term recall. J Personal Disord 1997; 11:19-33.

17. Green AH. Child sexual abuse. Immediate and long-term effects and intervention. J Am Acad Child Adolesc Psychiatry 1993; 32:890-902.

18. Sarwer DB, Crawford I, Durlak JA. The relationship between childhood sexual abuse and adult male sexual dysfunction. Child Abuse Neglect 1997; 21:649-655.

19. Sarwer DB, Durlak JA. Childhood sexual abuse as a predictor of adult female sexual dysfunction: a study of couples seeking sex therapy. Child Abuse Neglect 1996; 20:963-972.

20. Polusny MA, Follette VM. Long-term correlates of child sexual abuse: theory and review of the empirical literature. Appl Prevent Psychol 1995; 4:143-166.

21. Pribor EF, Dinwiddie SH. Psychiatric correlates of incest in childhood. Am J Psychiatry 1992; 149:52-56.

22. Smolak L, Murnen SK. A meta-analytic examination of the relationship between child sexual abuse and eating disorders. Int J Eat Disord 2002; 31:136-150.

23. Wonderlich SA, Brewerton TD, Jocic Z, Dansky BS, Abbott DW. Relationship of childhood sexual abuse and eat- 
ing disorders. I Am Acad Child Adolesc Psychiatry 1997; 36:1107-1115.

24. Pope HG Jr, Hudson JI. Is childhood sexual abuse a risk factor for bulimia nervosa? Am J Psychiatry 1992; 149:455-463.

25. Welch SL, Fairburn CG. Childhood sexual and physical abuse as risk factors for the development of bulimia nervosa: a community-based case-control study. Child Abuse Negl 1996; 20:633642.

26. Felitti VJ. Long-term medical consequences of incest, rape, and molestation. South Med J 1991; 84:328-331.

27. Springs FE, Friedrich WN. Health risk behaviors and medical sequelae of childhood sexual abuse. Mayo Clin Proc 1992; 67:527-532.

28. Felitti VJ. Childhood sexual abuse, depression, and family dysfunction in adult obese patients: a case control study. South Med J 1993; 86:732-736.

29. Felitti VJ, Anda RF, Nordenberg D, Williamson DF, Spitz AM, Edwards V, Koss MP, Marks JS. Relationship of childhood abuse and household dysfunction to many of the leading causes of death in adults. Am J Prev Med 1998; 14:245-258.

30. Dansky BS, Brewerton TD, Kilpatrick DG, O’Neil PM. The national women's study: relationship of victimization and posttraumatic stress disorder to bulimia nervosa. Int J Eat Disord 1997; 21:213-228.

31. Brewerton TD, O’Neil PM, Dansky BS, Kilpatrick DG. Links between morbid obesity, victimization, PTSD, major depression \& bulimia in a national sample of women (Abstract). Obes Res 1999; 7:56S.

32. Wiederman MW, Sansone RA, Sansone LA. Obesity among sexually abused women: an adaptive function for some? Women Health 1999; 29:89-100.

33. Grilo CM, Masheb RM. Childhood psychological, physical, and sexual maltreatment in outpatients with binge eating disorder. frequency and associations with gender, obesity, and eating-related psychopathology. Obes Res 2001; 9:320-325.

34. Striegel-Moore RH, Dohm F, Pike KM, Wilfley DE, Fairburn CG. Abuse, bullying, and discrimination as risk factors for binge eating disorder. Am J Psychiatry 2002; 159:1902-1907.

35. King KK, Clark MM, Pera V. History of sexual abuse and obesity treatment outcome. Addict Behav 1996; 21:283-290.

36. Wonderlich SA, Crosby RD, Mitchell JE, Roberts JA, Haseltine B, DeMuth G, Thompson KM. Relationship of childhood sexual abuse and eating disturbance in children. J Am Acad Child Adolesc Psychiatry 2000; 39:1277-1283.

37. Fichtner CG, O’Connor FL, Yeoh HS, Arora R, Crayton JR. Hypodensity of platelet serotonin uptake sites in posttraumatic stress disorder: associated clinical features. Life Sci 1995; 57:3744.

38. Maes M, Lin A, Verkerk R, Delmeire L, Gastel AV, Van der Planken M. Serotonergic and noradrenergic markers of post-trau- matic stress disorder with and without major depression. Neuropsychopharmacology 1999; 20:188-197.

39. Steiger H, Gauvin L, Israël M, Koerner N, Ng Ying Kin NM, Paris J. Association of serotonin and cortisol indices with childhood abuse in bulimia nervosa. Arch General Psychiatry 2001; 58:837-843.

40. Steiger H. Eating disorders and the serotonin connection: state, trait and developmental effects. Rev Psychiatr Neurosci 2004; 29:20-29.

41. Brewerton TD. Toward a unified theory of serotonin dysregulation in eating and related disorders. Psychoneuroendocrinology $1995 ; 20: 561-590$.

42. Blundell JE. Serotonin manipulations and the structure of feeding behavior. Appetite 1986; 7:39-56.

43. Loeb TB, Williams JK, Carmona JV, Riukin I, Wyatt GE, Chin D, Asuan-O'Brien A. Child sexual abuse: associations with the sexual functioning of adolescents and adults. Annu Rev Sex Res 2002; 13:307-345.

44. Tovee MJ, Hancock PJ, Mahmoodi S, Singleton BR, Cornelissen PL. Human female attractiveness: waveform analysis of body shape. Proc R Soc Lond B Biol Sci 2000; 269:22052213.

45. Sarwer DB, Grossbart TA, Didie ER. Beauty and society. Seminars Cutaneous Medical Surg 2003; 22:79-92.

46. Rand CS, Stunkard AJ. Psychoanalysis and obesity. J Am Acad Psychoanal 1977; 5:459-497.

47. Hooker D, Convisser E. Women's eating problems: an analysis of a coping mechanism. Personnel Guidance J 1983; 62:236-239.

48. Sarwer DB, Thompson JK. Obesity and body image disturbance. In: Wadden, TA, Stunkard, AJ (eds). Handbook of Obesity Treatment. Guilford Press: New York, 2002, pp. 447-464.

49. Clark MM, Ruggiero L, Pera V, Goldstein MG, Abrams DB. Assessment, classifications, and treatment of obesity: behavioral medicine perspective. In: Stoudemine, A, Fogel, BS (eds). Psychiatric Care of the Medical Patient. Oxford University Press, New York, 1993, pp. 903-926.

50. Weiner EJ, Stephen L. Sexual barrier weight: a new approach. In: Schwartz, MF, Cohn, L (eds). Sexual Abuse and Eating Disorders. Bruner/Mazel, New York, 1996, pp. 68-77.

51. Felitti VJ, Williams SA. Long term follow-up and analysis of over one hundred patients who have lost over 100 pounds. Permanente J 1998; 2:12-21.

52. Roman SE, Martin JL, Morris E, Herbison GP. Psychological defense styles in women who report childhood sexual abuse: a controlled community study. Am J Psychiatry 1999; 156:10801085 .

53. Bensley LS, Van Eenwyk J, Simmons KW. Self-reported childhood sexual and physical abuse and adult HIV-risk behaviors and heavy drinking. Am J Prev Med 2000; 18:151-158. 